\title{
Lateral Ventricular Meningiomas: Clinical Features, Radiological Findings and Long-Term Outcomes
}

\author{
Haibo Teng ${ }^{1} * *$ \\ Zhiyong Liu',* \\ Ouying Yan $^{2}$ \\ Wenbo $\mathrm{He}^{1}$ \\ Danyang Jie ${ }^{3}$ \\ Yuanwei Qie ${ }^{4}$ \\ Jianguo $\mathrm{Xu} \mathbb{D}^{\prime}$
}

'Department of Neurosurgery, West China Hospital, Sichuan University, Chengdu, Sichuan, People's Republic of China; ${ }^{2}$ Department of Radiation Oncology, The Affiliated Cancer Hospital of Xiangya School of Medicine, Central South University, Changsha, Hunan, People's Republic of China; ${ }^{3}$ Department of Neurosurgery, West China School of Medicine, West China Hospital, Sichuan University, Chengdu, Sichuan, People's Republic of China; ${ }^{4}$ Health Management Center, West-China Fourth Hospital, Sichuan University, Chengdu, Sichuan, People's Republic of China

*These authors contributed equally to this work
Correspondence: Jianguo $\mathrm{Xu}$ Department of Neurosurgery, West China Hospital, Sichuan University, Chengdu, People's Republic of China Email xujg@scu.edu.cn
Purpose: Lateral ventricle meningioma (LVM) is a rare type of intracranial meningioma, which has been rarely studied. It has different clinical features, imaging features, and longterm results from other locations. This study investigated the epidemiology, clinical characteristics and prognosis of LVM and comprehensively describes its characteristics.

Methods: This article analyzes the LVMs that were diagnosed pathologically in West China hospital between January 1, 2009 and July 1 2020. Demographic information, imaging characteristics and prognostic factors are discussed. Data analysis was performed using SPSS 23.0 and R version 3.5.3.

Results: We collected 7202 meningiomas and 195 LVMs (136 females; median age, 46 years; range, 5-81 years) were included in this study. Gross total resection was completed in 189 patients. The OS rate was $93.8 \%$, and the recurrence rate was 5.2\%. Multivariate regression analysis showed that $\operatorname{sex}(\mathrm{P}=0.01)$ and tumor size $(\mathrm{P}=0.018)$ were related to WHO grade. Postoperative KPS $(\mathrm{P}=0.003)$ was associated with OS. WHO grade $(\mathrm{P}=$ $0.025)$, extent of tumor resection $(\mathrm{P}<0.001)$, and hospital day $(\mathrm{P}=0.028)$ were associated with recurrence.

Conclusion: LVMs require long-term follow-up, individualized treatment, and follow-up strategies to be formulated according to the relevant risk factors.

Keywords: lateral ventricular meningiomas, imaging features, prognosis, recurrence

\section{Introduction}

Meningioma is the second most common primary tumor of the central nervous system with an annual incidence of $1.5-5.5$ per $100,000 .^{1,2}$ Intraventricular meningiomas (LVMs) are rare tumors that account for $0.5-3 \%$ of all intracranial meningiomas. ${ }^{3}$ Due to sufficient growth space, tumors are often already large when symptoms appear. Although surgical techniques continue to improve, some patients develop postoperative recurrence or metastasis. So far, there have been few reports and studies of $\mathrm{LVMs}^{3}$ and they are incomplete in terms of imaging and clinical data, as well as adequate and relevant risk prediction analyses. It is difficult to obtain tissues for histopathological diagnosis before the surgery; therefore, many LVMs are difficult to diagnose. Compared with other meningioma locations, lateral ventricle meningiomas have different characteristics. Neurologic symptoms typically are more delayed and tumors are quite large at initial diagnosis. ${ }^{4}$ Partial excision may lead to increased postoperative bleeding. ${ }^{5}$ Gross total resection is possible through a superior parietal lobule in most LVMs, but a report also showed that the elderly with left side lesion have a greater risk. ${ }^{6}$ An improved understanding of intraventricular meningiomas can help to diagnosis and apply 
appropriate treatments. Consequently, we have summarized all cases of LVM undergoing surgery and diagnosed in our hospital in the past 11 years, and conducted detailed statistics and analysis.

\section{Materials and Methods}

\section{Subject Selection}

This study was approved by the West China hospital Ethics Committee. A total of 7202 patients were histologically confirmed meningioma at the West China Hospital between January 1, 2009 and July 1, 2020 and 195 LVMs were included in the study. Patients were identified by HIS (Hospital Information System). The inclusion criteria for the research are that LVM was confirmed by postoperative pathology and we excluded cases with incomplete clinical information. The tumor sections were reviewed by two expert pathologists and graded histologically according to The 2016 World Health Organization Classification of Tumors of the Central Nervous System.

We obtained the following clinical information from HIS including demographic information, clinical features and surgical management. The data of preoperative magnetic resonance imaging (MRI) and the computed tomography (CT) scan were examined via PACS (Picture Archiving and Communication Systems). The Karnofsky Performance Scale (KPS) was used to assess the quality of life. Preoperative and postoperative KPS (pre-KPS and post-KPS) at discharge was evaluated by two authors (Haibo Teng and Zhiyong Liu). Clinic and imaging follow-up examinations were performed at the time of 1,6 , 12 months after surgery. In addition, patients were followed-up by telephone every 1 or 2 years and recorded the KPS scores. Hospital day (HOD) was defined as the number of days between the day of surgery and discharge.

In terms of imaging data, tumor size was defined as the largest single dimension recorded from the measurement of the MRI/CT preoperative imaging. Where postoperative imaging was missing or unavailable in the PACS, we relied on the surgeon's observation based on the operative record to require the extent of resection. After excluding unqualified or missing preoperative imaging data, we collected a total of 118 patients finally. Signal intensity (SI) of the tumor on T1- and T2weighted and flair MRI was is divided into mixedintense, hypo-intense, iso-intense and hyper-intense. The shape of tumor was defined base on the solid component appearance and categorized as regular and irregular forms. On T2-weighted imaging, the presence of cerebral edema is judged as a hyperintensity near the tumor. Clear tumor-brain boundary is defined as hypo-intense on T1weighted imaging and hyperintense on T2-weighted imaging. For the SI on the images of enhancement, the levels were homogeneous enhancement (uniformly enhancing tissue in $>90 \%$ of the tumor) and heterogeneous enhancement (well-enhanced and less-enhanced portions were mixed).

Regarding extent of resection (EOR), we reviewed both the operative records and postoperative MRI imaging reports from each patient. The EOR was categorized as gross total removal (GTR), subtotal removal (STR) based on the comparison of preoperative and postoperative magnetic resonance conditions. We obtained EOR data of 3 patients without postoperative MRI through Surgical Record. Data analysis was performed using SPSS 23.0 and $\mathrm{R}$ version 3.5.3.

\section{Result}

\section{Patient Population}

During the 11 years of study research (2009-2020), a total of 7202 meningioma patients underwent surgical procedures in West China Hospital, including 195 cases (59 male and 136 female) of LVM. The basic information is provided in Table 1. The mean and median follow-up time were $64(0-143)$ and 61 months. The mean and median ages of patients were 45 years (range 5-81 years) and 46 years. Eighty-eight patients were on the right side, 96 were on the left side, and 10 were on both sides. Eight patients had received previous tumor-related treatment before enrolment (surgery in 1 patient, radiation in 6 patients and surgery followed by radiation treatment in 2 patients). The median duration of symptoms prior to admission was 60 months (range 0-123), including headache and dizziness $(\mathrm{n}=122,68.2 \%)$, visual impairment $(\mathrm{n}=16,8.2 \%)$, movement disorders $(\mathrm{n}=11,11.6 \%)$, epilepsy $(\mathrm{n}=11$, $11.6 \%$ ) and others like memory loss, numbness, tinnitus $(\mathrm{n}=25,12.8 \%)$. Besides this, 10 asymptomatic patients accounted for $5.1 \%$ of all patients. The median preoperative KPS score was 90 (range 20-100). Among the entire cohort, 110 patients (56.4\%) were pre-KPS scores $\geq 80$ and 85 patients $(43.6 \%)$ were pre-KPS scores $<80$. In addition, 12 patients $(6.2 \%)$ with periprocedural complications were found in the cohort (10 hydrocephalus and 2 cerebral hernia). 
Table I Patient Characteristics

\begin{tabular}{|c|c|}
\hline & Value \\
\hline Median follow-up period, mos (range) & $61(0-143)$ \\
\hline Median age, yrs (range) & $46(5-8 I)$ \\
\hline Sex, F/M & $136 / 54$ \\
\hline Side, $r t / l t / b o t h$ & $88 / 96 / 10$ \\
\hline Mediantumorsize, mm (range) & $43(3-120)$ \\
\hline Previou streatments & 9 \\
\hline Surgery & 1 \\
\hline Radiationtherapy & 6 \\
\hline Surgery followed by radiation therapy & 2 \\
\hline Median preop symptom duration, mos (range) & $60(0-3700)$ \\
\hline \multicolumn{2}{|l|}{ Preop symptom } \\
\hline Headache and dizziness & $122(68.2 \%)$ \\
\hline Visual impairment & $16(8.2 \%)$ \\
\hline Movement disorders & II (II.6\%) \\
\hline Epilepsy & II (II.6\%) \\
\hline Others & $25(12.8 \%)$ \\
\hline Asymptomatic & $10(5.1 \%)$ \\
\hline \multicolumn{2}{|l|}{ Resection } \\
\hline GTR & 189 \\
\hline STR & 6 \\
\hline \multicolumn{2}{|l|}{ Pathology } \\
\hline WHO grade I & $145(74.4 \%)$ \\
\hline WHO grade II & $44(22.5 \%)$ \\
\hline WHO grade III & $6(3.1 \%)$ \\
\hline Radiographic recurrence & $12(6.2 \%)$ \\
\hline \multicolumn{2}{|l|}{ Treatment after recurrence } \\
\hline Surgery followed by radiation therapy & 3 \\
\hline Radiationtherapy & 5 \\
\hline Conservative Treatment & 4 \\
\hline
\end{tabular}

\section{Radiological and Pathological Characteristics}

The radiological information of 118 patients with complete neuroimaging data of all 195 LVM patients in our hospital available are presented in Table 2 . The great majority of IVMs had irregular shape ( $\mathrm{n}=87,73.7 \%)$, clear boundary $(\mathrm{n}=102,86.4 \%)$ and no cystic $(\mathrm{n}=107,90.7 \%)$. The mean and median tumor size were $46 \mathrm{~mm}$ and $43 \mathrm{~mm}$ (range 3$120 \mathrm{~mm}$ ), with 46 tumors $(38.9 \%)>40 \mathrm{~mm}$ and 72 tumors $(61.1 \%) \leq 40 \mathrm{~mm}$ in diameter. The edema ranged from 0 to $49 \mathrm{~mm}$, with 26 tumors $(22.1 \%) \geq 30 \mathrm{~mm}$, and 92 tumors $(77.9 \%)<30 \mathrm{~mm}$. The cases of mixed-intense on the T1-
Table 2 The MRI Features in the II8 Cases of LVMs

\begin{tabular}{|c|c|}
\hline & Value \\
\hline Tumor size $(\mathrm{mm})$ & $43(3-120)$ \\
\hline \multicolumn{2}{|l|}{ Shape } \\
\hline Regular & $3 \mathrm{I}(26.3 \%)$ \\
\hline Irregular & 87 (73.7\%) \\
\hline \multicolumn{2}{|l|}{ Boundary } \\
\hline Clear & $102(86.4 \%)$ \\
\hline Ambiguous & $16(13.6 \%)$ \\
\hline Edema (mm) & $2(0-49)$ \\
\hline \multicolumn{2}{|l|}{ Cystic } \\
\hline No & $107(90.1 \%)$ \\
\hline Yes & II (I0.2\%) \\
\hline \multicolumn{2}{|l|}{ TI-weighted } \\
\hline Mixed & 44 (37.2\%) \\
\hline Нyро- & $10(8.5 \%)$ \\
\hline Iso- & $33(28.0 \%)$ \\
\hline Hyper- & 31 (26.3\%) \\
\hline \multicolumn{2}{|l|}{ T2-weighted } \\
\hline Mixed & 77 (65.3\%) \\
\hline Нyро- & $6(5.1 \%)$ \\
\hline Iso- & 13 (II.0\%) \\
\hline Hyper- & 22 (I8.6\%) \\
\hline \multicolumn{2}{|l|}{ Flair-weighted } \\
\hline Mixed & 80 (67.9\%) \\
\hline Нуро- & $5(4.2 \%)$ \\
\hline Iso- & 7 (5.9\%) \\
\hline Hyper- & $26(22.0 \%)$ \\
\hline \multicolumn{2}{|l|}{ Enhancement } \\
\hline Heterogenous & 81 (68.6\%) \\
\hline Homogenous & 37 (3I.4\%) \\
\hline
\end{tabular}

weighted $\quad(\mathrm{n}=44, \quad 37.2 \%), \quad$ T2-weighted images $(n=77,65.3 \%)$ and flair-weighted images $(n=80,67.9 \%)$ were observed in the majority of the LVMs (Figure 1). In MRI enhancement sequence, 37 cases $(31.4 \%)$ were homogenous and 81 cases $(68.6 \%)$ were heterogeneous. All 195 patients had complete pathological data included for this research. According to the 2016 WHO classification, the pathological results were divided into WHO I grade $(\mathrm{n}=145,74.4 \%)$, WHO II grade $(\mathrm{n}=44,25.5 \%)$ and WHO III grade $(n=6, n=3.1 \%)$. The univariate analysis of WHO grade was summarized in Table 3. It indicated that sex $(\mathrm{P}=0.005)$, tumor size $(\mathrm{P}=0.02)$, preoperative KPS $(\mathrm{P}=0.04)$, and edema degree $(\mathrm{P}=0.02)$ were associated with higher pathological grade. Results of multivariate 


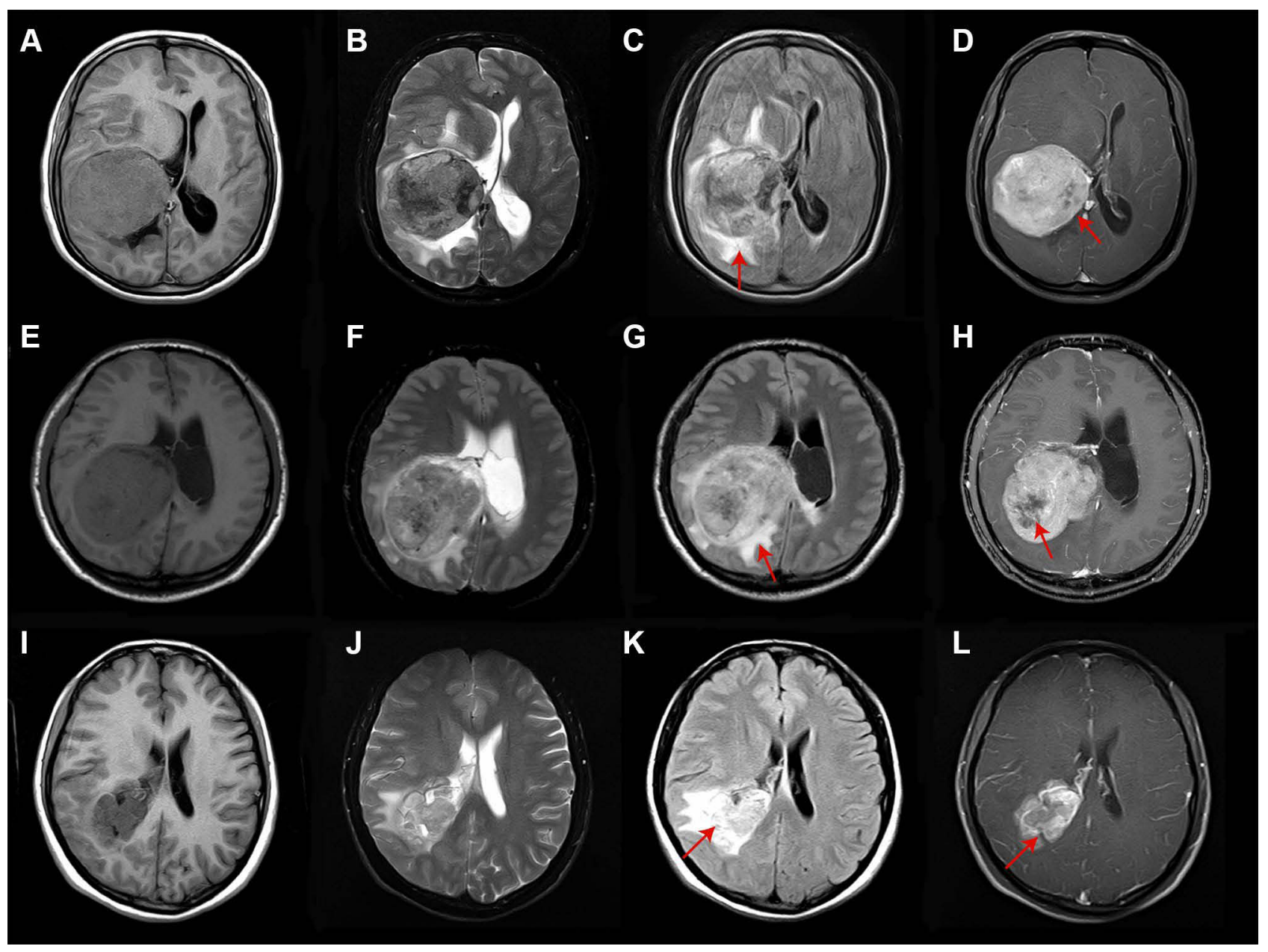

Figure I MRI images of typical WHO I (A-D), WHO II (E-H) and WHO III (I-L) of LVM. Most MRI images show mixed-intense and heterogeneous enhancement. Typical low-grade LVM is relatively more regular and clearer boundary, while high-grade LVM has more obvious peritumoral edema and tissue infiltration. Heterogeneous enhancement and perifocal edema in which MRI sequences are indicated by arrows.

analyses for association with $\operatorname{sex}(\mathrm{P}=0.01)$ and tumor size $(\mathrm{P}=0.018)$ are shown in Table 3 . There was no statistically significant difference $(\mathrm{p}>0.05)$ in all radiological feature including SI and enhancement.

\section{Treatment and Long-Term Outcomes}

After a median follow-up duration of 46 months (range 0146 months), the most recent follow-up data were obtained in 186 patients $(95.4 \%)$ but were lost in 9 patients $(4.6 \%)$. The overall survival rate of LVMs patients in 11 years was $93.8 \%$. By the end of the follow-up period, twelve patients $(6.2 \%)$ developed a recurrence following the surgery and 12 patients $(6.2 \%)$ died. The Kaplan-Meier survival and recurrence curve is shown in Figure 2. The preoperative and postoperative KPS score was no statistically significant difference $(\mathrm{P}=$ $0.108)$ in all patients; however, 86 patients (44.1\%) with KPS $\leq 80$ points showed significant improvement of KPS postoperatively $(\mathrm{P}<0.01)$ (Figure 3$)$. In general, most of the patients' symptoms have improved significantly, and part or all of their life and work can be resumed after the operation. A gross total resection (GTR) was completed in almost all patients $(\mathrm{n}=189,97.2 \%)$, and a subtotal resection only in 6 patients $(2.8 \%)$. GTR has better PFS (progression-free survival) than STR. The curve of univariate analysis related to OS and recurrence is shown in Figures 4 and 5. The result of multivariate Cox regression analysis is summarized in Figure 6. Only postoperative KPS $(\mathrm{P}=0.003)$ was positively associated with the OS of LVMs in the analysis (HR 9.06, 95\% CI: 2.15-38.10). Additionally, the multivariable analyses indicated that the following factors were related to tumor recurrence: WHO grade $(\mathrm{P}=0.025 ; \mathrm{HR}=4.407$; $\mathrm{CI}$ : $1.20=16.19)$, the extent of tumor resection $(\mathrm{P}<0.001$; $\mathrm{HR}=0.05$; CI: $0.01-0.24$ ) and HOD (hospital day) $(\mathrm{P}=0.028 ; \mathrm{HR}=4.75 ; \mathrm{CI}: 1.18-19.06)$. 
Table 3 Univariate and Multivariate Analysis of WHO Grade

\begin{tabular}{|c|c|c|c|c|c|}
\hline & Low-Grade & High-Grade & $\mathbf{P}$ & HR & $\mathbf{P}$ \\
\hline Sex & & & 0.005 & $\begin{array}{c}0.273 \\
(0.102-0.73)\end{array}$ & 0.01 \\
\hline Male & 36 & 23 & & & \\
\hline Famle & 109 & 27 & & & \\
\hline Tumor size & & & 0.02 & $\begin{array}{c}5.222 \\
(1.336-20.416)\end{array}$ & 0.02 \\
\hline$\geq 40 \mathrm{~mm}$ & 69 & 13 & & & \\
\hline$<40 \mathrm{~mm}$ & 70 & 30 & & & \\
\hline Age & & & 0.91 & & \\
\hline Pre-KPS & & & 0.04 & 1.334 & 0.58 \\
\hline & & & & $(0.484-3.679)$ & \\
\hline$>80$ & 87 & 22 & & & \\
\hline$\leq 80$ & 58 & 28 & & & \\
\hline Shape & & & 0.18 & & \\
\hline Regular & 27 & 4 & & & \\
\hline Irregular & 66 & 21 & & & \\
\hline Boundary & & & 0.68 & & \\
\hline Clear & 81 & 21 & & & \\
\hline Ambiguous & 12 & 4 & & & \\
\hline Edema & & & 0.05 & 1.749 & 0.30 \\
\hline & & & & $(0.6 \mid 4-4.983)$ & \\
\hline$\geq 30 \mathrm{~mm}$ & 17 & 9 & & & \\
\hline$<30 \mathrm{~mm}$ & 70 & 16 & & & \\
\hline Cystic & & & 0.60 & & \\
\hline No & 85 & 22 & & & \\
\hline Yes & 8 & 3 & & & \\
\hline TI-weighted & & & 0.52 & & \\
\hline Mixed & 32 & 12 & & & \\
\hline Нyро- & 8 & 2 & & & \\
\hline Iso- & 26 & 7 & & & \\
\hline Hyper- & 27 & 4 & & & \\
\hline T2-weighted & & & 0.30 & & \\
\hline Mixed & 58 & 19 & & & \\
\hline Нуро- & 6 & 0 & & & \\
\hline Iso- & 12 & 1 & & & \\
\hline Hyper- & 17 & 5 & & & \\
\hline Flair-weighted & & & 0.64 & & \\
\hline Mixed & 62 & 18 & & & \\
\hline Нyро- & 5 & 0 & & & \\
\hline Iso- & 6 & I & & & \\
\hline Hyper- & 20 & 6 & & & \\
\hline Enhancement & & & 0.37 & & \\
\hline Heterogenous & 62 & 19 & & & \\
\hline Homogenous & 31 & 6 & & & \\
\hline
\end{tabular}




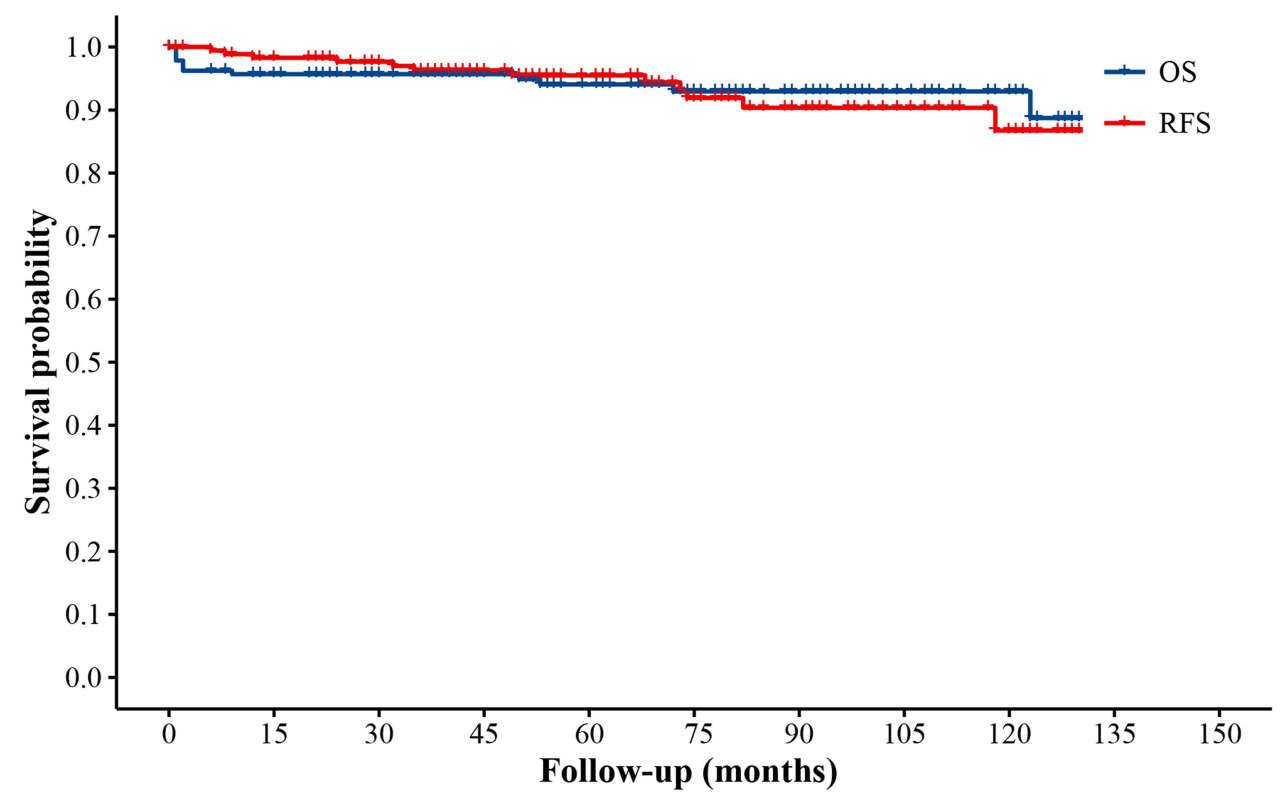

Figure 2 Kaplan-Meier survival curve illustrates the overall survival, and progression-free survival of 195 LVM patients.

A

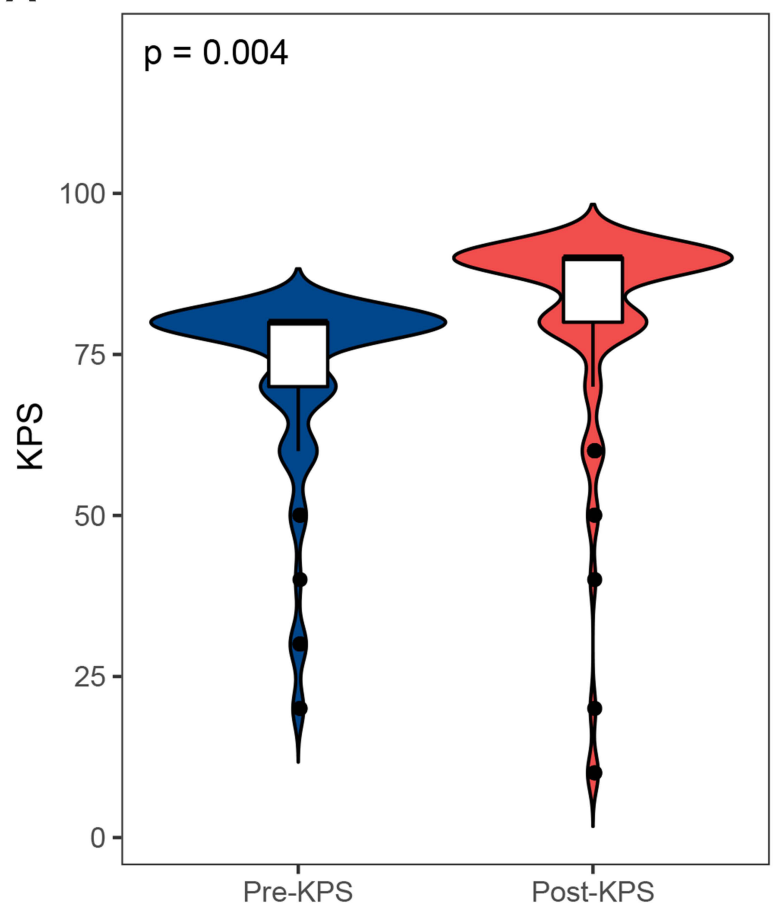

B

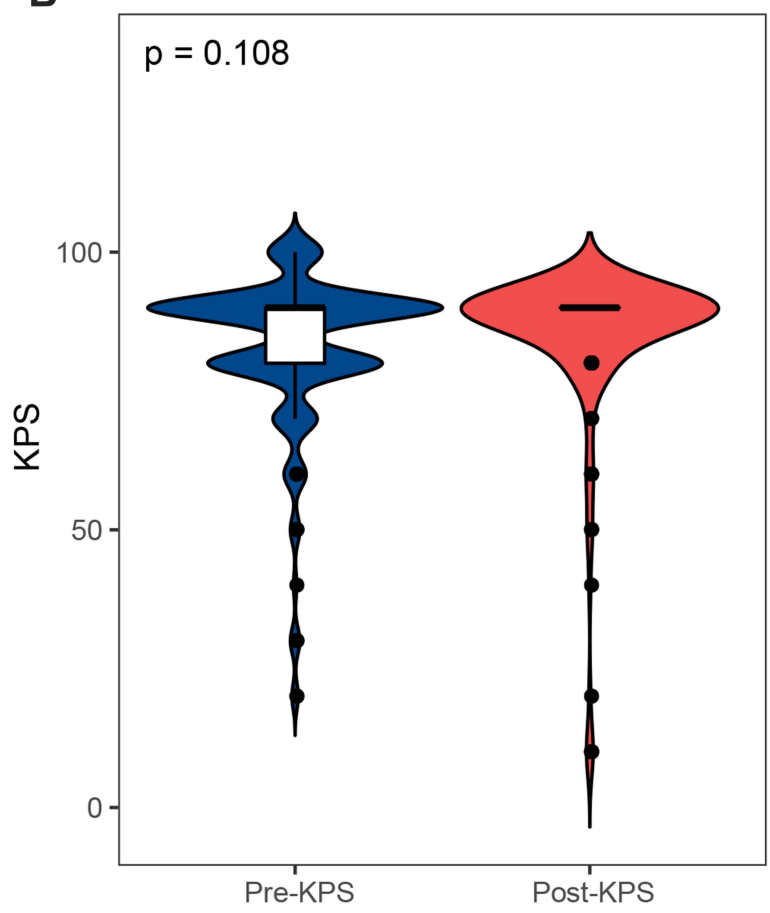

Figure 3 (A) Violin plot illustrating a comparison between the pre- and post-KPS of 85 patients with preoperative KPS score $\leq 80$. (B) Violin plot illustrating a comparison between the pre- and post-KPS of all 195 patients.

\section{Discussion}

\section{Incidence and Clinical Characteristics}

LVM is rare, accounting for only $0.5-3 \%$ of all meningiomas, ${ }^{7}$ and has different clinical features and pathological conditions compared to meningiomas in other locations. LVMs are defined as tumors originating from the arachnoid cells in the mesenchymal stroma of the choroid plexus and velum interpositum in the intraventricular location. ${ }^{8,9,10}$ LVMs are extremely uncommon, resulting in inclusion of a small sample size in previous 

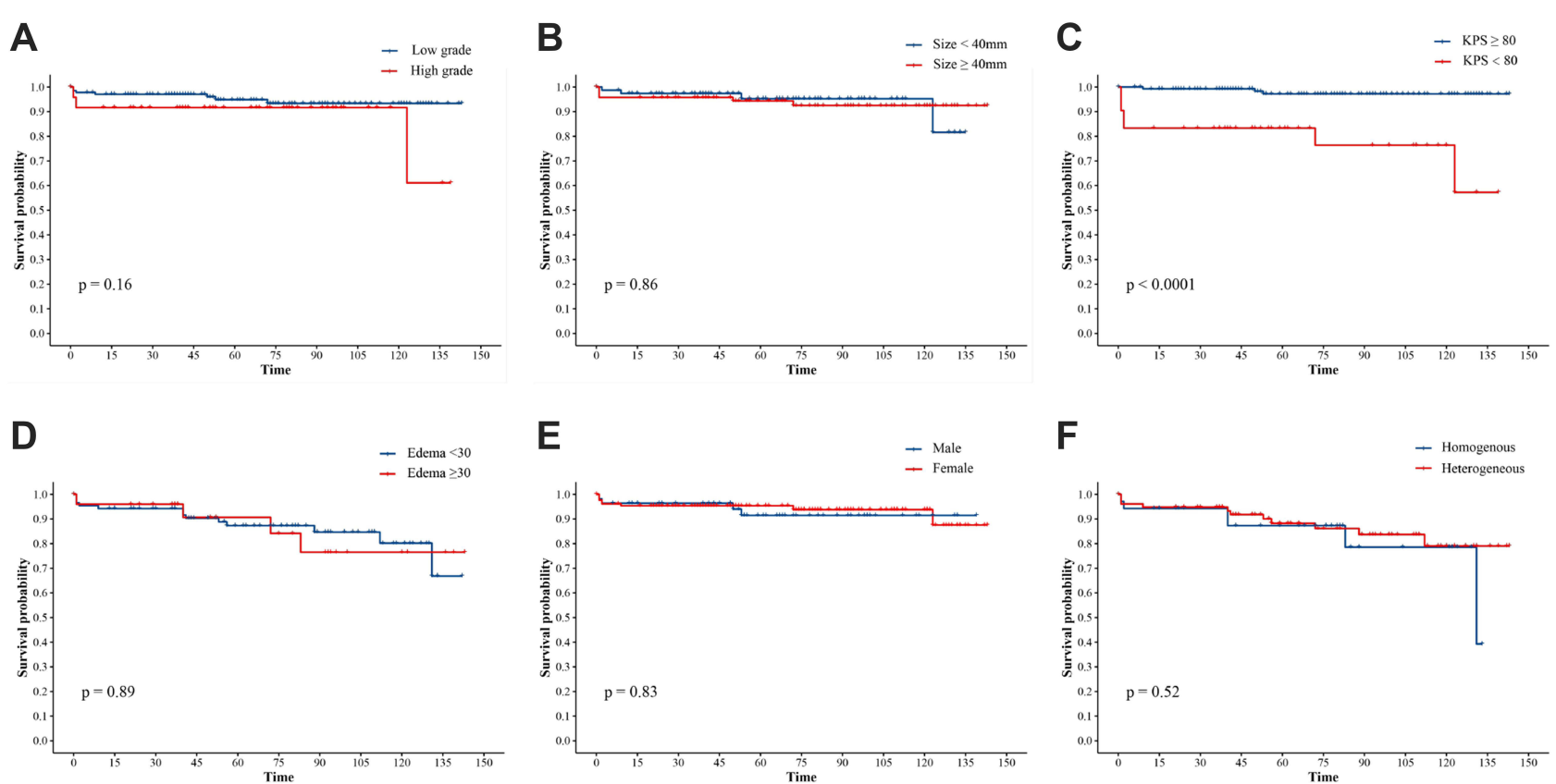

Figure 4 Kaplan-Meier survival analysis illustrating risk factors for OS. A comparison between the OS based on the WHO grade (A), the tumor size (B), the pre-KPS of patients (C), the extent of edema (D), the gender (E), the MRI enhancement (F).
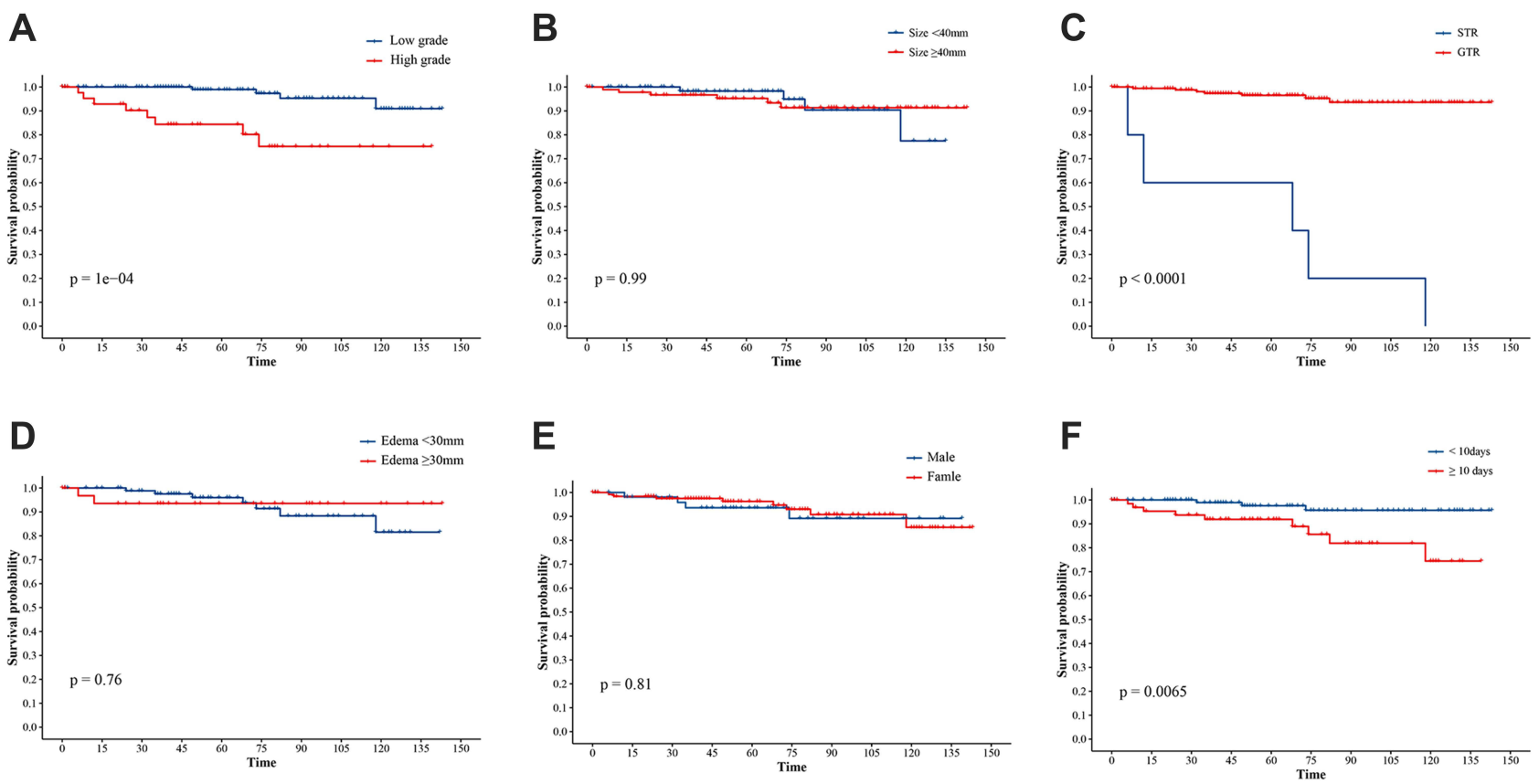

Figure 5 Kaplan-Meier survival analysis illustrating risk factors for recurrence. A comparison between the OS based on the WHO grade (A), the tumor size (B), the extent of resection (C), the extent of edema (D), the gender (E), the hospital day (F).

studies. To the best of our knowledge, our study is the largest case series of LVMs, with the longest follow-up time, in a single center. We systematically reviewed and analyzed the baseline characteristics, neuroimaging features, pathology data, and the survival outcomes, including both relapse and OS of IVMs, to form a more comprehensive understanding of IVMs.

In 11 years, 7202 patients with meningiomas were diagnosed in our hospital. LVM accounts for $2.7 \%(n=195)$, which is similar to previous reports in the literature. Among 
A

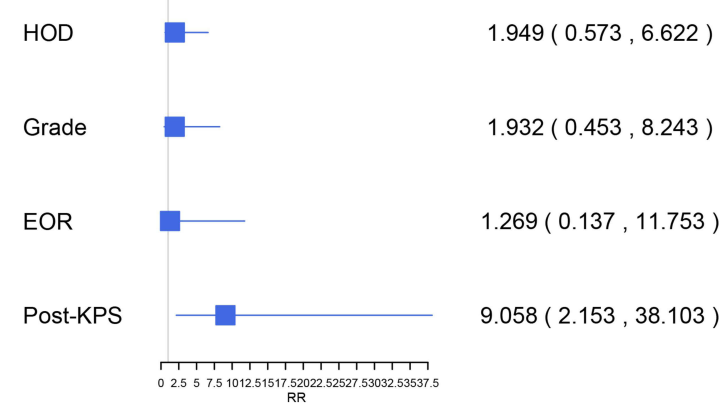

B

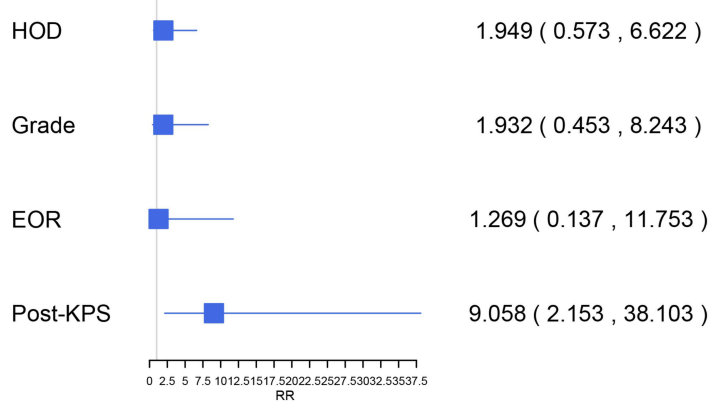

Figure 6 The result of multivariate Cox regression analysis. HR for risk of OS (A) and recurrence (B) the blue squares indicate the HR or OR values, and error bars represent $95 \% \mathrm{Cls}$.

195 cases of LVM, female patients had a higher incidence (F/ $\mathrm{M}=2.3: 1$ ), which is similar to previous study. ${ }^{3}$ The contrasting sex distribution may occur because of a difference in progesterone levels between females and males. However, the proportion of high-grade meningiomas in male patients was higher than in female patients $(\mathrm{P}=0.005)$, and the ratio of incidence in males to females for high-grade meningiomas was the same $(\mathrm{F} / \mathrm{M}=1: 1)$. Similar to the meningiomas in other locations, the incidence of LVMs on the left was higher than that on the right side. A specific reason for this difference is not known. ${ }^{10}$ The age distribution of LVMs is different from that of other meningiomas in general. Previous studies have reported that the age of onset of meningiomas is approximately 60 years. ${ }^{11}$ Nevertheless, since IVMs are more symptomatic than meningiomas in other locations, we found that the age of onset of LVMs was earlier. The average age of IVMs was 44.9 years, including elderly ( 34 cases $\geq 60$ years, $17.4 \%$ ), adults (150 cases, $77.0 \%$ ), and children (11 cases $\leq 20$ years, $5.6 \%$ ). Furthermore, unlike meningioma in other locations, the clinical manifestations of LVM are relatively silent, and mild symptoms often last for a long time. Most patients visit the hospital because of persistent headaches or vision problems. This may be due to the anatomy of the lateral ventricle and the mass effect, which also leads to a longer pre-hospital course. Therefore, the tumor usually appears relatively large in volume at the time of LVM diagnosis.

\section{Radiological and Pathological Characteristics}

The diagnosis of LVM was mainly performed using MRI. Pathological diagnosis remains a gold standard for LVM. The imaging findings and pathological features are presented in Table 2. Kshettry et al have reported that the overall proportion of WHO I, II, and III intracranial meningiomas were $94.6 \%, 4.2 \%$, and $1.2 \%$, respectively. ${ }^{12}$ In our cohort, we had a higher proportion of high-grade LVMs, especially WHO grade II (22.5\%) tumors. Due to lack of studies on a large population at a global scale, we cannot judge whether the pathological grade is associated with race and region. Our analysis has demonstrated that sex, tumor size, and edema were predictive factors for high-grade LVMs on a univariate analysis (Table 3). We found that high-grade LVMs were larger than WHO grade I. Similar to the findings of previous studies, ${ }^{13-15}$ edema was found to be a prognostic factor for the pathological grade of meningiomas by univariate analysis. NaKano et al have suggested that the invasive pattern of the tumorbrain interface was related to brain edema of meningiomas. ${ }^{15}$ Go et al have pointed out that edema fluid flows into the brain tissue from the tumor, following cortical disruption by tumor invasion. ${ }^{13,14}$ However, this relationship disappeared after controlling for confounders using a multivariate analysis.

It has been reported by Whittle et al that meningiomas on MRI are iso- to hypointense on T1-weighted imaging and hyperintense on T2-weighted imaging with strong homogeneous enhancement. Simultaneously, heterogeneous tumor enhancement has been indicated in high-grade meningiomas. ${ }^{16-18}$ However, there have been no imagingrelated studies on LVM in the past. In our cohort, all the T1, T2 and Flair-weighted LVMs in MRI were dominated by mixed-intense. Most enhancement MRI of LVMs, including low-grade LVMs, usually appear as heterogeneous enhancement. In the subsequent analysis, we did not find a significant relationship between MRI signals and WHO grade. This could 
be due to the duration of LVMs and relatively more free space to expand, eventually unraveling heterogeneous enhancement.

High-grade LVMs $(n=31)$ showed a significantly irregular shape compared to low-grade LVMs $(n=87)$, although we found no statistical significance between the tumor shape and WHO grade in the analysis. Lin et al considered that unclear boundary of the tumor was a predictive factor of high-grade meningiomas ${ }^{18}$ and a feature of aggressive biological behavior. ${ }^{19,20}$ However, our data revealed no significant relationship between the boundary and grade of LVMs. It is possible that despite the high grade of LVMs, the tumor has ample space to grow before invasion of the surrounding brain tissue, which results in a clear boundary. Therefore, we propose that using imaging features of overall meningiomas to judge the grade of LVMs is not accurate. In the present study, our analysis identified only sex and tumor size as independent predictors to distinguish low- from high-grade LVMs.

\section{Treatment and Prognosis}

Similar to the other locations of meningioma, patients with LVMs have good long-term survival. There are two main reasons for the death of LVMs in our study cohort. The first reason is postoperative complications $(n=7)$ within two months after the surgery. The second reason is mainly due to disease progression or non-tumor-related death $(n=5)$. We found that only post-KPS was an independent factor associated with OS of LVMs in the multivariate analysis. Therefore, improvements in surgical techniques are important for the treatment of LVMs. Surgery is still the main mode of treatment for LVMs, and GTR continues to be the primary target of surgery. The rate of GTR in our cohort was $96.9 \%$. STR was chosen mainly because the tumor invaded the surrounding tissues, and to avoid damaging the white matter and causing serious complications. There was no statistically significant difference between pre- and post-KPS in all LVM patients after surgery, in our hospital. This may be due to the large space in the lateral ventricle. In most patients, the tumors were found through mild symptoms; therefore, surgery was performed at an early stage. However, in patients with a KPS $\leq 80$, the KPS after the operation was significantly better than that before the operation $(\mathrm{P}<0.001)$.

In addition, even WHO grade I LVMs also have a risk of long-term recurrence. Twelve patients (6.2\%) in our cohort had relapses. Multivariate analysis showed that WHO classification, EOR (extent of resection), and
HOD significantly correlated with recurrence. In 12 cases with recurrence, high-grade LVMs had an absolute advantage $(n=8)$, and the average recurrence time was significantly shorter than that of WHO I LVMs. A highgrade LVM indicates a greater possibility of recurrence, which may be due to pathological parameters related to the aggressiveness of the tumor. Pathological markers such as E-Ca, Ki67, and $\beta$-catenin are thought to be related to recurrence. ${ }^{21}$ Proliferation parameters such as MIB-1 LI have also been proposed to predict the recurrence of meningioma. ${ }^{22-25}$ Ros-Sanjuan et al advocated that total resection was the only significant factor associated with recurrence, ${ }^{26}$ and furthermore, Simpson proved that the degree of resection grading system he proposed was related to tumor recurrence. ${ }^{27}$ However, some scholars believe that the Simpson classification cannot be used as a predictive indicator. ${ }^{28} \mathrm{Vo} 3 \mathrm{~K}$ et al proved that Simpson grade is not related to the recurrence of intraventricular meningioma. ${ }^{29}$ Due to the uniqueness of lateral ventricle anatomy and improvements at surgical technique, the GTR rate is higher than the other locations. We found that LVMs still have a high recurrence rate in the cohort (especially high-grade LVMs). The present confirmed that the EOR is related to RFS, EOR alone is not sufficient to predict the recurrence. In some studies, Simpson classification combined with pathological classification was used to predict the recurrence. ${ }^{30}$ However, in our study, in addition to these two factors, HOD was also a predictor of recurrence. We believe that for patients with longer HOD, the operation is more difficult, and it is more intuitive to consider that the tumor is more aggressive, which may lead to more postoperative complications. Therefore, we believe that a combination of these three factors will be more effective in predicting the recurrence of LVMs. Unterberger et al indicated that surgery + adjuvant radiotherapy (ART) can reduce the recurrence of high-grade meningiomas in their review. ${ }^{31}$ This is consistent with the results of most studies. ${ }^{32-35}$ Studies have also shown that the prognosis of patients undergoing ART is worse. ${ }^{36-38}$ However, there are no randomized controlled trials to prove this. In our cohort, 6 cases of 10 patients who received postoperative radiotherapy had a recurrence. This may be due to higher tumor grade in the patients who chose radiotherapy in our hospital (6 cases of WHO III). Therefore, we cannot prove the correlation between postoperative radiotherapy and recurrence rate. 


\section{Limitations}

This study has some limitations. First, our study is retrospective, and bias is possible. Second, we lacked complete imaging data for all the LVM patients, which could lead to deviations in statistical analysis. Third, we require multicenter studies and larger samples to obtain more accurate results on the characteristics of LVMs. Fourth, many patients refuse ART, so there are deficiencies in the conclusion of postoperative radiotherapy. Finally, low-grade LVMs require more follow-up time to determine survival and recurrence.

\section{Conclusion}

LVM is rare and has different characteristics from other subtypes of meningioma. We conducted the largest singlecenter retrospective study to date. Our research shows that the age at onset of LVMs is younger, and the course of the disease is more occult. The pathological grade is only related to age and size, and it is unreliable to judge the WHO grade through imaging. Patients with LVMs have a high GTR rate and a good survival rate, but the longterm recurrence rate is still high, especially, for high-grade LVMs. The survival rate is only related to the postoperative KPS, while the recurrence rate is related to the WHO grade, EOR, and HOD. Therefore, individualized treatment and follow-up plans should be developed for patients with LVMs with related high risks.

\section{Data Sharing Statement}

The datasets generated during and/or analysed during the current study are available from the corresponding author on reasonable request.

\section{Ethics Approval}

All procedures performed in studies involving human participants were in accordance with the ethical standards of the institutional and/or national research committee and with the 1964 Helsinki Declaration and its later amendments or comparable ethical standards. The study was approved by the ethics Committee of the West China hospital (No. 2020(990)).

\section{Consent to Participate}

Informed consent was obtained from all individual participants and the parents of all children included in the study.

\section{Author Contributions}

Haibo Teng: Conception and design, methodology and investigation, data analysis and interpretation, study supervision, and writing and review. Zhiyong Liu: Data collection, Data analysis and interpretation and writing and review. Ouying Yan: Data analysis and interpretation and writing and review. Wenbo He: Data analysis and interpretation and review. Danyang Jie and Yuanwei Qie: Data analysis and interpretation and review. Jianguo $\mathrm{Xu}$ : Conception and design, methodology and investigation, data analysis and interpretation, study supervision, and writing and review. All authors made substantial contributions to conception and design, acquisition of data, or analysis and interpretation of data; took part in drafting the article or revising it critically for important intellectual content; agreed to submit to the current journal; gave final approval of the version to be published; and agree to be accountable for all aspects of the work. Haibo Teng and Zhiyong Liu are co-first authors.

\section{Funding}

This work was supported by Key research and development project of science and technology department of Sichuan Province (2019YFS0392/3); 1.3.5 project for disciplines of excellence, West China Hospital, Sichuan University (ZYJC18007).

\section{Disclosure}

Authors have no conflict of interest to declare.

\section{References}

1. Claus E, Bondy M, Schildkraut J, Wiemels J, Wrensch M, Black P. Epidemiology of intracranial meningioma. Neurosurgery. 2005;57 (6):1088-1095. doi:10.1227/01.NEU.0000188281.91351.B9

2. Chen W, Magill S, Englot D, et al. Factors associated with pre- and postoperative seizures in 1033 patients undergoing supratentorial meningioma resection. Neurosurgery. 2017;81(2):297-306. doi:10.1093/neuros/nyx001

3. Pereira BJA, de Almeida AN, Paiva WS, de Aguiar PH, Teixeira MJ, Marie SK. Natural history of intraventricular meningiomas: systematic review. Neurosurg Rev. 2020;43(2):513-523. doi:10.1007/s10143-0181019-0

4. Menon G, Nair S, Sudhir J, Rao R, Easwer H, Krishnakumar K. Meningiomas of the lateral ventricle - a report of 15 cases. $\mathrm{Br}$ J Neurosurg. 2009;23(3):297-303. doi:10.1080/02688690902721862

5. Lyngdoh B, Giri P, Behari S, Banerji D, Chhabra D, Jain V. Intraventricular meningiomas: a surgical challenge. $J$ Clin Neurosci. 2007;14(5):442-448.

6. Criscuolo G, Symon L. Intraventricular meningioma. A review of 10 cases of the National Hospital, Queen Square (1974-1985) with reference to the literature. Acta Neurochir. 1986;83:83-91. 
7. Li Z, Li H, Jiao Y, et al. Clinical features and long-term outcomes of pediatric intraventricular meningiomas: data from a single neurosurgical center. Neurosurg Rev. 2018;41(2):525-530. doi:10.1007/ s10143-017-0884-2

8. Civit T, Taillandier L, Baylac F. Chordoid meningioma. J Neurosurg. 1998;89(4):686-687.

9. Baumgartner J, Sorenson J. Meningioma in the pediatric population. J Neurooncol. 1996;29(3):223-228.

10. Bhatoe H, Singh P, Dutta V. Intraventricular meningiomas: a clinicopathological study and review. Neurosurg Focus. 2006;20 (3):E9.

11. Magill ST, Young JS, Chae R, Aghi MK, Theodosopoulos PV, McDermott MW. Relationship between tumor location, size, and WHO grade in meningioma. Neurosurg Focus. 2018;44(4):E4. doi:10.3171/2018.1.FOCUS17752

12. Kshettry VR, Ostrom QT, Kruchko C, Al-Mefty O, Barnett GH, Barnholtz-Sloan JS. Descriptive epidemiology of World Health Organization grades II and III intracranial meningiomas in the United States. Neuro Oncol. 2015;17(8):1166-1173. doi:10.1093/ neuonc/nov069

13. Go K, Kamman R, Wilmink J, Mooyaart E. A study on peritumoral brain edema around meningiomas by MRI and contrast CT. J Acta Neurochirurgica Supplementa. 1994;60:365-368.

14. Go K, Wilmink J, Molenaar WJN. Peritumoral brain edema associated with meningiomas. Neurosurgery. 1988;23(2):175-179.

15. Nakano T, Asano K, Miura H, Itoh S, Suzuki S. Meningiomas with brain edema: radiological characteristics on MRI and review of the literature. Clin Imaging. 2002;26(4):243-249.

16. Whittle IR, Smith C, Navoo P, Collie D. Meningiomas. Lancet. 2004;363(9420):1535-1543. doi:10.1016/S0140-6736(04)16153-9

17. Kim E, Kim S, Kim H, Jeon P, Kim K, Byun H. Intraventricular meningiomas: radiological findings and clinical features in 12 patients. Clin Imaging. 2009;33(3):175-180.

18. Lin B, Chou K, Kao H, et al. Correlation between magnetic resonance imaging grading and pathological grading in meningioma. $J$ Neurosurg. 2014;121(5):1201-1208.

19. Nakasu S, Nakajima M, Matsumura K, Nakasu Y, Handa J. Meningioma: proliferating potential and clinicoradiological features. Neurosurgery. 1995;37(6):1049-1055.

20. Takeguchi T, Miki H, Shimizu T, et al. Prediction of tumor-brain adhesion in intracranial meningiomas by MR imaging and DSA. Magn Reson Med Sci. 2003;2(4):171-179.

21. Ye W, Ding-Zhong T, Xiao-Sheng Y, Ren-Ya Z, Yi L. Factors related to the post-operative recurrence of atypical meningiomas. Front Oncol. 2020;10:503.

22. Aguiar P, Tsanaclis A, Tella O, Plese J. Proliferation rate of intracranial meningiomas as defined by the monoclonal antibody MIB-1: correlation with peritumoural oedema and other clinicoradiological and histological characteristics. Neurosurg Rev. 2003;26(3):221-228.

23. Kudryavtsev G, Kudryavtseva L, Mikhaleva L, Solovieva N, Babichenko I. [Immunohistochemical study of Ki-67, p53 and Notch1 expressions in prostate cancer of different grades]. Arkhiv Patologii. 2020;82(5):42-49. Russian.
24. Kasuya H, Kubo O, Tanaka M, Amano K, Kato K, Hori T. Clinical and radiological features related to the growth potential of meningioma. Neurosurg Rev. 2006;29(4):293-296; discussion 296-297.

25. Kim Y, Ketter R, Steudel W, Feiden W. Prognostic significance of the mitotic index using the mitosis marker anti-phosphohistone H3 in meningiomas. Am J Clin Pathol. 2007;128(1):118-125.

26. Ros-Sanjuan A, Iglesias-Moroño S, Carrasco-Brenes A, BautistaOjeda D, Arraez-Sanchez M. Atypical meningiomas: histologic and clinical factors associated with recurrence. World Neurosurg. 2019;125:e248-e256.

27. Simpson D. The recurrence of intracranial meningiomas after surgical treatment. J Neurol Neurosurg Psychiatry. 1957;20(1):22-39. doi:10.1136/jnnp.20.1.22

28. Schwartz T, McDermott M. The Simpson grade: abandon the scale but preserve the message. J Neurosurg. 2020;1:1-8.

29. Voß K, Spille D, Sauerland C, et al. The Simpson grading in meningioma surgery: does the tumor location influence the prognostic value? J Neurooncol. 2017;133(3):641-651.

30. Oya S, Kawai K, Nakatomi H, Saito N. Significance of Simpson grading system in modern meningioma surgery: integration of the grade with MIB-1 labeling index as a key to predict the recurrence of WHO grade I meningiomas. J Neurosurg. 2012;117(1):121-128.

31. Unterberger A, Nguyen T, Duong C, Kondajji A, Kulinich D, Yang I. Meta-analysis of adjuvant radiotherapy for intracranial atypical and malignant meningiomas. $J$ Neurooncol. 2021;152(2):205-216. doi:10.1007/s11060-020-03674-7

32. Chen W, Magill S, Wu A, et al. Histopathological features predictive of local control of atypical meningioma after surgery and adjuvant radiotherapy. $J$ Neurosurg. 2018;130(2):443-450.

33. Aghi M, Carter B, Cosgrove G, et al. Long-term recurrence rates of atypical meningiomas after gross total resection with or without postoperative adjuvant radiation. Neurosurgery. 2009;64(1):56-60; discussion 60

34. Zhi M, Girvigian M, Miller M, et al. Long-term outcomes of newly diagnosed resected atypical meningiomas and the role of adjuvant radiotherapy. World Neurosurg. 2019;122:e1153-e1161.

35. Cain S, Smoll N, Van Heerden J, Tsui A, Drummond K. Atypical and malignant meningiomas: considerations for treatment and efficacy of radiotherapy. J Clin Neurosci. 2015;22(11):1742-1748.

36. Bagshaw H, Burt L, Jensen R, et al. Adjuvant radiotherapy for atypical meningiomas. J Neurosurg. 2017;126(6):1822-1828.

37. Masalha W, Heiland D, Franco P, et al. Atypical meningioma: progression-free survival in 161 cases treated at our institution with surgery versus surgery and radiotherapy. J Neurooncol. 2018;136 (1):147-154

38. Hammouche S, Clark S, Wong A, Eldridge P, Farah J. Long-term survival analysis of atypical meningiomas: survival rates, prognostic factors, operative and radiotherapy treatment. Acta Neurochir. 2014;156(8):1475-1481.
Cancer Management and Research is an international, peer-reviewed open access journal focusing on cancer research and the optimal use of preventative and integrated treatment interventions to achieve improved outcomes, enhanced survival and quality of life for the cancer patient.
The manuscript management system is completely online and includes a very quick and fair peer-review system, which is all easy to use. Visit http://www.dovepress.com/testimonials.php to read real quotes from published authors. 